\title{
How I treat hepatitis $C$ virus infection in patients with hematologic malignancies
}

\author{
Dr. Rasha Kamel, MD \\ Lecturer of Internal Medicine, \\ Department of Hematology and Bone Marrow transplantation, \\ Ain Shams University
}

\section{Introduction}

$\mathrm{HCV}$ is a hepatotropic, lymphotropic virus affecting over 180 million people, all over the world by chronic infection. ${ }^{1}$ In Egypt, the prevalence rate of HCV infected individuals was 872,000 ( $15 \%$ of the population) in 2013 , with an estimated incidence of newly infected 125,000 viremic individuals each year ${ }^{2}$, the rate which is considered as one of the highest prevalence rates of HCV worldwide. ${ }^{3}$

Management of HCV infection is more challenging in patients with hematologic malignancies than in patients without cancer because of a higher rate of progression of fibrosis, more rapid development of cirrhosis, increased viral titers, worse outcome, and exclusion from some cancer and/or antiviral treatments. Elimination of HCV from infected patients with hematologic malignancies has the potential for virologic, hepatic, and oncologic benefits (Table 1$){ }^{4}$

Table (1) Benefits of HCV treatment in patients with hematologic malignancies (5)

\section{Infectious and hepatic}

1. Prevention of long-term complications such as liver cirrhosis and end-stage liver disease

2. Prevention of hepatocellular carcinoma as a primary or secondary cancer in patients with chronic HCV infection

3. Improvement of long-term survival

4. Treatment of extrahepatic manifestations (cryoglobulinemia, fatigue)

\section{Oncologic}

1. Allowing patients access to multiple clinical trials of cancer chemotherapies, including trials of agents with hepatic metabolism

2. Prevention of some HCV-associated hematologic malignancies (e.g. non-Hodgkin lymphoma) or decrease of the relapse rate of those malignancies

3. 3. Cure of selected HCV-associated hematologic malignancies without chemotherapy 
Among patients infected with HCV, those with hematologic malignancies, and especially patients who have undergone $\mathrm{HCT}$, have a more rapid rate of fibrosis progression and a higher risk of developing cirrhosis than patients without cancer. Among patients with cancer, HCV screening and early diagnosis, assessment of liver fibrosis, and elimination of HCV will likely improve long-term outcomes. Groups for whom HCV screening is recommended include candidates for HCT, patients with hematologic malignancies and other cancer patients as recommended in patients without cancer. HCV screening is recommended before starting selected chemotherapy agents (e.g., rituximab, alemtuzumab) and before enrolment on clinical trials with investigational antineoplastic agents. Discovery of HCV infection in a patient newly diagnosed with a hematologic malignancy should prompt virologic tests and fibrosis assessments (Table 2).

Table (2): Initial evaluation of HCV-infected patients with hematologic malignancies. ${ }^{7}$

\begin{tabular}{|c|c|c|c|}
\hline $\begin{array}{l}\text { History and clinical } \\
\text { findings }\end{array}$ & Laboratory tests & Virologic tests & Imaging/staging studies \\
\hline $\begin{array}{l}\text { History } \\
\text { - Alcohol abuse } \\
\text { - Metabolic risk factors } \\
\text { - Vaccination status } \\
\text { against HAV and HBV } \\
\text { Physical examination } \\
\text { Symptoms/signs of } \\
\text { cirrhosis }\end{array}$ & $\begin{array}{l}\text { Routine } \\
\text { - Complete blood } \\
\text { count, AST, ALT, total } \\
\text { bilirubin,alkaline } \\
\text { phosphatase, } \\
\text { albumin, PT/PTT/INR, } \\
\text { BUN, creatinine } \\
\text { Others } \\
\text { - } \text { Alpha-fetoprotein } \\
\text { - GGT } \\
\text { - Cryoglobulins } \\
\text { - Selected cases } \\
\text { - Interleukin } 28 \mathrm{~B} \\
\text { polymorphism }\end{array}$ & $\begin{array}{l}\text { HCV } \\
\text { - HCV-RNA } \\
\text { quantitation } \\
\text { HCV genotype } \\
\text { Co-infections } \\
\text { - } \text { Anti-HAV } \\
\text { - HBsAg } \\
\text { - Anti-HBs } \\
\text { - Anti-HBc } \\
\text { Selected cases } \\
\text { - HCV riV } \\
\text { testing resistance }\end{array}$ & $\begin{array}{l}\text { Imaging } \\
\text { Abdominal sonography } \\
\text { or computed tomography } \\
\text { Noninvasive markers of } \\
\text { fibrosis } \\
\text { - Vibration-controlled } \\
\text { transient elastography } \\
\text { Serum fibrosis panel } \\
\text { Pathology } \\
\text { - Liver biopsy }\end{array}$ \\
\hline
\end{tabular}


In HCV-infected cancer patients, ALT levels should be monitored during chemotherapy because immunocompromised cancer patients can experience acute exacerbation of chronic HCV infection (also known as hepatitis flare), which is indicated by a significant elevation of serum ALT levels over the baseline level. In a retrospective study of $308 \mathrm{HCV}$-infected patients treated for cancer, $11 \%$ developed an acute exacerbation of chronic HCV infection, defined as a 3-fold or greater increase in serum ALT level from baseline in the absence of infiltration of the liver by cancer, use of hepatotoxic medications, blood transfusion within 1 month of elevation of ALT level, or other hepatic viral infection. Acute exacerbation of HCV infection during chemotherapy prompted clinicians to discontinue chemotherapy in nearly half of infected patients. The diagnostic work-up to confirm acute exacerbation of HCV infection must consider a range of possibilities and take into account that multiple causes of liver inflammation can be present at the same time. In a patient who has undergone HCT, additional diagnoses to consider include the hepatic presentation of graft-versus-host disease (GVHD), infection with other viruses, drug-induced liver injury, and hypoxic hepatitis. ${ }^{8}$

HCV-infected cancer patients undergoing immunosuppressive therapy may also experience increased HCV replication (also known as HCV reactivation), which has been defined as an increase in HCV-RNA viral load of at least $1 \log 10 \mathrm{IU} / \mathrm{mL}$ over baseline after chemotherapy or immunosuppressive therapy (chronically infected patients have stable HCVRNA levels that may vary by approximately $0.5 \log 10 \mathrm{IU} / \mathrm{mL}$ ). The increased replication of $\mathrm{HCV}$ and the resulting high blood titers of virus appear to be associated with a more indolent course than hepatitis B virus (HBV) reactivation; there are only a few reports of deaths associated with increased HCV replication, some of them related to development of fibrosing cholestatic hepatitis C. ${ }^{9}$

In general, in patients with hematologic malignancies and HCV infection, ALT level should be evaluated at baseline and periodically during chemotherapy or immunosuppressive therapy to identify unusual cases of more severe hepatocellular injury or fibrosing cholestatic hepatitis C. Routine monitoring of HCV RNA is not recommended; however, HCV RNA should be measured in all patients at entry into care, and viral load should be monitored in patients receiving $\mathrm{HCV}$ treatment according to standard-of-care management for patients without cancer. ${ }^{4}$

Eradication of HCV may normalize liver function, allowing access to drugs that would otherwise be contraindicated, including agents with hepatic metabolism. SVR would prevent $\mathrm{HCV}$ reactivation and hepatic flare after chemotherapy, thus avoiding discontinuation or dose reduction of chemotherapy and possibly the risk of hepatic decompensation during cancer care. Longer-term benefits would include prevention of progression to cirrhosis, reduction in 
the risk of second primary cancers (hepatocellular carcinoma and NHL), and improved survival of patients with these secondary cancers. ${ }^{10}$

The advent of DAAs has rendered IFN-containing regimens obsolete for treatment of $\mathrm{HCV}$ infection, and the benefits of DAA therapy outweigh the risks in HCV-infected patients with hematologic malignancies (Table 1). Contraindications to treatment with DAAs in HCV infected patients with hematologic malignancies are shown in Table 3.

Table (3): Subgroups of HCV-infected patients with hematologic malignancies for whom DAA therapy is contraindicated. ${ }^{4}$

- Patients with uncontrolled hematologic malignancy or other comorbidities associated with a life

- expectancy of less than 12 months owing to non-liver-related conditions

- Patients with moderate or severe hepatic impairment (Child-Pugh class B or C)

- Pregnant women and men whose female partners are pregnant if ribavirin is considered

- Patients with major drug-drug interactions with chemotherapy or immunosuppressive agents that

- cannot be temporarily discontinued

- Patients with known hypersensitivity or intolerance to drugs used to treat HCV

To improve the response to HCV therapy and avoid development of viral resistance, DAA therapy should not be interrupted or given intermittently. DAA-based regimens can be completed in 8 to 12 weeks in most patients with selected cases requiring up to 24 weeks of treatment, enabling eradication of HCV during chemotherapy-free periods. Severe adverse effects and hematologic toxic effects are uncommon (occurring in fewer than $5 \%$ of patients) in cancer patients receiving IFN-free or ribavirin-free DAA-containing regimens. ${ }^{11}$

Simultaneous administration of chemotherapy and DAAs is not recommended. However, preliminary data in a small group of cancer patients, including some with hematologic malignancies, showed that concomitant chemotherapy and DAA therapy is feasible. Because we have only limited clinical understanding of drug-drug interactions and chemotherapy tolerability in HCV-infected patients, simultaneous therapies should be used with caution. ${ }^{12}$

\section{HCV and lymphoma}

Evidence that HCV infection may be causal in the development of lymphoma is based on several cases in which regression of lymphoma followed elimination of HCV. Most patients 
with HCV-associated NHL have mild liver disease at the time of lymphoma diagnosis, suggesting that DAA therapy should be initiated as early as possible after diagnosis of HCV infection, regardless of liver disease status, to eradicate HCV infection and thus, prevent extrahepatic manifestations such as NHL. Evidence for this strategy comes from a Japanese study in which the annual incidence of lymphoma was compared between 501 HCV-infected patients who had never received interferon (IFN)-based therapy and 2708 HCV-infected patients who had received IFN. The subsequent risk of lymphoma was approximately 7 times higher in patients with persistent HCV infection as in patients with IFN-induced SVR. Among patients whose therapy eliminated HCV, there were no cases of lymphoma development by 15 years. $^{13}$

Furthermore, several case series have shown regression of indolent lymphoma in HCVinfected patients treated with antiviral drugs. Current National Comprehensive Cancer Network guidelines on splenic marginal zone lymphoma recommend treatment of HCV without chemotherapy as first-line therapy for HCV-infected patients. The benefit of giving DAA therapy without chemotherapy as first-line therapy may extend to patients with other types of HCV-associated NHL (e.g., diffuse large B-cell lymphoma) or patients with HCVassociated NHL undergoing hematopoietic cell transplant (HCT). ${ }^{14}$

In a study of $30 \mathrm{HCV}$-infected cancer patients, including 15 with hematologic malignancy, who achieved a SVR before cancer therapy, no patient had viral relapse after cancer therapy. The cancer therapies were as follows: rituximab, cyclophosphamide, cisplatin, 5-fluorouracil, doxorubicin, melphalan, bortezomib, fludarabine, paclitaxel, sorafenib, lenalidomide, and vincristine, (some patients received combination therapy). These findings indicate that HCV infection is curable in cancer patients and the risk of HCV recurrence is low once SVR has been achieved, even after chemotherapy-related immune suppression. These findings also emphasize the importance of identification and treatment of HCV infection before initiation of chemotherapy. Patients with evidence of fibrotic and necroinflammatory liver disease require monitoring of liver disease progression even when they have achieved a SVR. ${ }^{15}$

\section{HCV and HSCT}

A 2015 report from the American Society for Blood and Marrow Transplantation Task Force extensively covers diagnosis and management of HCV infection in hematopoietic cell donors and HCT candidates and recipients. Virtually all HCV-infected donors will transmit virus to recipients. Case reports of HCV-infected marrow donors treated with IFN or ribavirin demonstrate that clearance of HCV from the bloodstream prevents passage of virus, an effect that does not 
require a SVR, just temporary cessation of viremia, during which marrow or peripheral blood is harvested. DAAs are very effective in clearing HCV and work rapidly; thus, when the best HLAmatched donor is HCV-infected, treatment with DAAs should be instituted as early as possible. Most infected donors will attain undetectable HCV-RNA within 4 weeks of initiation of DAA therapy. ${ }^{16}$

If no uninfected HLA-matched donor is available and if time does not permit treatment of the infected donor to eliminate HCV from the infusion product, the use of an HCV-infected hematopoietic inoculum into an HCV-uninfected recipient is not contraindicated. The risk of dying from the underlying hematologic malignancy without HCT far outweighs the risk of acquiring potentially curable HCV. However, the donor should be assessed for advanced liver disease, extrahepatic manifestations of HCV (e.g., NHL), and coinfections (e.g., HIV) that might contraindicate donation. Using an HCV-infected sibling donor is preferable to an HLA matched unrelated donor, as the natural history of HCV infection in allografted patients is usually benign in the early years following HCT. After transplant, DAA therapy can be given to the recipient. For most patients with early hematologic malignancy, survival after HCT with hematopoietic cells donated by an HLA-matched sibling is superior to survival after an unrelated donor transplant. ${ }^{17}$

Fibrosing cholestatic hepatitis, a rare and potentially fatal complication of HCT characterized by periportal fibrosis, ballooning degeneration of hepatocytes, prominent cholestasis, and paucity of inflammation related to a high intracellular load of either HBV or HCV and viral protein. Fibrosing cholestatic hepatitis has been described after HCT, organ transplant, and some chemotherapy regimens. In an HCT recipient, fibrosing cholestatic hepatitis $C$ must be differentiated from other manifestations of cholestatic liver injury (e.g., GVHD, drug-induced liver injury, and cholestasis of infection). ${ }^{18}$

In both the liver transplant and HCT settings, use of mycophenolate mofetil has been linked to development of fibrosing cholestatic hepatitis $C$; thus, this drug should probably not be used in HCV-infected patients. In the HCT setting, DAAs against HCV would likely reduce the burden of intracellular virus and reduce the mortality rate of fibrosing cholestatic hepatitis $C$, as has been observed with successful DAA therapy of fibrosing cholestatic hepatitis $C$ in the liver transplant setting. ${ }^{19}$

When possible, DAA therapy for HCV-infected HCT candidates should be completed before HCT. If DAA therapy cannot be completed until after HCT, DAA therapy can be deferred until after immune reconstitution except in patients who develop fibrosing cholestatic hepatitis $\mathrm{C}$ and probably on cases of severe $\mathrm{HCV}$ reactivation post HCT. ${ }^{20}$ 
Drawback of deferring DAA therapy for HCV infection until after HCT is the propensity of DAAs for drug interactions, including altered disposition of several drugs commonly used in HCT patients. The drugs most impacted by DAAs are components of conditioning regimens, calcineurin inhibitors, and sirolimus, but review of the isoenzymes responsible for drug metabolism suggests that many of the drugs used in supportive care are similarly affected by DAAs. Current databases (http://www.hep-druginteractions.org and Lexicomp online) should be consulted along with the product prescribing information to ensure the safety of delivering DAAs together with medications such as acid reducers, antidepressants, antihypertensive, phosphodiesterase inhibitors, novel oral anticoagulants, macrolide antibiotics, and HMG CoA inhibitors. Some experts advocate waiting for 6 months after HCT to start DAA therapy, to allow tapering of immunosuppressive agents and GVHD prophylaxis; this practice might result in higher SVR rates and avoid drug-drug interactions with calcineurin inhibitors. The majority of HCV-infected HCT recipients do not have an adverse course in the years following HCT despite greatly increased titers of circulating virus following conditioning therapy. ${ }^{\mathbf{2 1}}$

It is not currently known whether the high rates of HCV clearance with DAAs in the general population and in organ transplant patients can be replicated in HCT patients in the early post-HCT period, as full immune reconstitution does not occur until more than 1 year after allogeneic HCT and both immunosuppressive therapy and GVHD will delay return of immunity. Preliminary data show that DAAs are safe and effective (SVR rate, $85 \%$ ) in HCVinfected HCT recipients. ${ }^{22}$

The choice of DAA regimen should be guided by several factors (e.g., the patient's prior antiviral treatment, HCV genotype, and degree of liver disease) and should be individualized after thorough assessment for potential hematologic toxic effects and drugdrug interactions. Several articles have been recently published on drug interactions with DAAs. Recommended dosage adjustments for patients with renal impairment are now available. For currently approved DAAs in 2016, no dose adjustments are necessary for patients with liver dysfunction, including those with decompensated cirrhosis. This issue have to be re-visited with each new DAA that is approved; and HCV guidelines and the manufacturer's package insert should be consulted. ${ }^{23}$

\section{Cirrhosis and hematological malignancy}

Dosing of drugs (including chemotherapy) in patients with cirrhosis is an inexact science, in part because of the complexity of drug disposition in the liver, which depends on hepatic perfusion, extraction, metabolism, excretion, and differences in protein binding of individual drugs. No single method allows estimation of the pharmacokinetics and pharmacodynamics of a 
drug in an individual patient with liver dysfunction. Dose adjustments are often necessary in patients with cholestatic liver injury, but adjustments are usually not necessary in patients with chronic HCV infection, except in the case of certain high-dose myeloablative conditioning regimens for $\mathrm{HCT}$, for which the risk of fatal sinusoidal obstruction syndrome is almost 10 times as high in patients with chronic HCV infection as in patients without HCV infection. The most accurate method of dose adjustment is therapeutic drug monitoring, in which the dose is personalized in real time according to an individual patient's clearance of a drug to achieve the target plasma exposure. ${ }^{24}$

Another method for dose adjustment involves triaging cirrhotic patients into ChildTurcotte-Pugh classes A, B, or C. For many chemotherapy drugs, pharmacokinetic information for patients with Child-Turcotte- Pugh class A or B cirrhosis is available from the drug manufacturer; such information is based on US Food and Drug Administration and European Medicines Agency guidance on determining the pharmacokinetics of study drugs in patients with impaired hepatic function. ${ }^{25}$

If the patient in this case were scheduled for HCT, the questions would be as follows:

What conditioning regimen could this patient with cirrhosis tolerate? Should DAA therapy be attempted before transplant? Is mycophenolate mofetil contraindicated as GVHD prophylaxis? High-dose myeloablative regimens containing sinusoidal endothelial cell toxins (cyclophosphamide, etoposide, thiotepa, melphalan, gemtuzumab ozogamicin, and total body irradiation $>12 \mathrm{~Gy}$ ) have been largely abandoned in patients with chronic hepatitis (HCV infection, nonalcoholic steatohepatitis, or alcoholic hepatitis) in favor of less liver-toxic regimens. If there is an imperative to use a CY-based regimen in a cirrhotic patient, the dose should be $90 \mathrm{mg} / \mathrm{kg}$ to $100 \mathrm{mg} / \mathrm{kg}$ (instead of $120 \mathrm{mg} / \mathrm{kg}$ ), dose-adjusted if possible, and either separated in time from $\mathrm{BU}$ or given first in order (e.g., $\mathrm{CY} /$ targeted $\mathrm{BU}$ ). ${ }^{\mathbf{2 6}}$

Reduced intensity regimens may avoid sinusoidal injury, but cirrhotic patients remain at risk for liver failure from GVHD, infection-related cholestasis, hypoxic hepatitis, tumor infiltration, and drug-induced liver injury resulting from drugs used in supportive care, including herbal therapies. Ascitic fluid should be drained prior to administration of hydrophilic drugs such as fludarabine and methotrexate ${ }^{52}$ and if mycophenolate mofetil is used, it should be dose-adjusted if the serum albumin level is low. Supportive care should include ursodiol for prevention of cholestatic liver injury, antibiotics to prevent bacterial translocation during neutropenia, and attention to portal pressures and hepatorenal syndrome. ${ }^{27}$ 
$\mathrm{HCV}$ is a risk factor for drug-induced liver injury from some drugs, and there are case reports of increased viral titers and more severe HCV infection after recovery of immunity following immunosuppressive chemotherapy. A cirrhotic patient would be at greater risk than someone without cirrhosis for worsening HCV-related liver inflammation. Treatment of HCVinfected cirrhotic patients with DAAs can be successful in achieving a SVR, but should probably be deferred until the course of hematologic malignancy is clear. ${ }^{28}$

Table (4): DAAs used to treat HCV in 2016 and their most common side effects. ${ }^{29}$

\section{Sofosbuvir}

- Fatigue, headaches

\section{Simeprevir}

- Fatigue, headaches, nausea, rash (including photosensitivity), pruritus

\section{Daclatasvir}

- Fatigue, headaches, anemia, nausea

Ombitasvir-Paritaprevir-Ritonavir and Dasabuvir

- Fatigue, nausea, pruritus, other skin reactions (e.g. rash, erythema, eczema), insomnia and asthenia

\section{Ledipasvir-Sofosbuvir}

- Fatigue, headache and asthenia

\section{Elbasvir-Grazoprevir}

- Fatigue, headache, nausea, anemiac

\section{Sofosbuvir-Velpatasvir}

- Headache, fatigue, anemia, nausea, headache, insomnia, and diarrhea.

\section{Conclusions}

HCV is now curable by DAAs in most patients, including those with hematologic malignancies. Elimination of HCV from infected patients offers potential virologic, hepatic, and oncologic benefits.

$\mathrm{HCV}$ infection should not contraindicate cancer therapy, and patients with chronic HCV infection and hematologic malignancies should not be excluded from clinical trials of chemotherapy or antiviral therapies. However, hepatologists and infectious diseases specialists with experience in treating HCV should participate in the diagnostic work-up, monitoring, and treatment of infected patients. 
In general, the DAA combinations recommended for cancer patients mimic those used for patients without cancer. DAAs used to treat HCV in 2016 and their most common side effects are shown in table 4. The optimal therapy for HCV infected patients with cancer is evolving rapidly and will continue to evolve as new DAAs are approved and as more studies are reported.

\section{Reference:}

1. Durand T, Di Liberto G, Colman H, Cammas A, Boni S, Marcellin P, et al. Occult infection of peripheral B cells by hepatitis $C$ variants which have low translational efficiency in cultured hepatocytes. Gut 2010;59:934-42.

2. Waked I, Doss W, El-Sayed MH, Estes C, Razavi H, Shiha G, et al. The current and future disease burden of chronic hepatitis C virus infection in Egypt. Arab J Gastroenterol 2014;15:45-52.

3. Sievert W, Altraif I, Razavi HA, Abdo A, Ahmed EA, Alomair A, et al. A systematic review of hepatitis C virus epidemiology in Asia, Australia and Egypt. Liver Int. 2011;31(Suppl. 2):61-80.

4. American Association for the Study of Liver Diseases (AASLD) and the Infectious Diseases Society of America (IDSA). HCV Guidance: Recommendations for testing $m$, and treating hepatitis $C$. http://www.hcvguidelines.org. Accessed July 11, 2016.

5. Torres HA, Mahale P, Blechacz B, et al. Effect of hepatitis $C$ virus infection in patients with cancer: addressing a neglected population. J Natl Compr Canc Netw. 2015;13(1):41-50.

6. Mallet V, van Bömmel F, Doerig C, et al. Management of viral hepatitis in patients with haematological malignancy and in patients undergoing haemopoietic stem cell transplantation: recommendations of the 5th European Conference on Infections in Leukaemia (ECIL-5). Lancet Infect Dis 2016;16:606-17.

7. Torres HA, McDonald GB. How we treat hepatitis $\mathrm{C}$ virus infection in patients with hematologic malignancies. blood-2016-05-718643

8. Mahale $\mathbf{P}$, Kontoyiannis DP, Chemaly RF, et al. Acute exacerbation and reactivation of chronic hepatitis $\mathbf{C}$ virus infection in cancer patients. J Hepatol. 2012; 57(6):1177-85.

9. McGovern BH, Birch CE, Bowen MJ, et al. Improving the diagnosis of acute hepatitis C virus infection with expanded viral load criteria. Clin Infect Dis. 2009; 49(7):1051-60.

10. Miyatake H, Kobayashi Y, Iwasaki Y, et al. Effect of previous interferon treatment on outcome after curative treatment for hepatitis C virus-related hepatocellular carcinoma. Dig Dis Sci. 2012;57(4):1092-101.

11. Asselah T, Boyer N, Saadoun D, Martinot-Peignoux M, Marcellin P. Direct-acting antivirals for the treatment of hepatitis $\mathrm{C}$ virus infection: optimizing current IFN-free treatment and future perspectives. Liver Int. 2016;36(Suppl 1):47-57.

12. Mahale $\mathbf{P}$, Kyvernitakis A, Kantarjian $\mathbf{H}$, et al. Concomitant use of chemotherapy and antivirals in hepatitis C virus infected cancer patients. The Liver Meeting $2015^{\circledR}$ 66th Annual Meeting of the American Association for the Study of Liver Diseases; 2015 November 13-17; San Francisco, CA. . p. Hepatology. 2015: 60(1)suppl; abstr 1160. 
13. Torres HA, Mahale P. Most patients with HCV-associated lymphoma present with mild liver disease: a call to revise antiviral treatment prioritization. Liver Int. 2015; 35(6):1661-4.

14. National Comprehensive Cancer Network (NCCN). Splenic Marginal Zone Lymphoma. Version 1, 2016.

15. Mahale P, Okhuysen PC, Torres HA. Does chemotherapy cause viral relapse in cancer patients with hepatitis C infection successfully treated with antivirals? Clin Gastroenterol Hepatol. 2014;12(6):1051-1054.

16. Hsiao HH, Liu YC, Wang HC, et al. Hepatitis C transmission from viremic donors in hematopoietic stem cell transplant. Transpl Infect Dis. 2014; 16(6):1003-6.

17. Pasquini M, Zhu X. Current use and outcome of hematopoietic stem cell transplantation CIBMTR summary slides, 2014.

18. Ceballos-Viro J, Lopez-Picazo JM, Perez-Gracia JL, et al. Fibrosing cholestatic hepatitis following cytotoxic chemotherapy for small-cell lung cancer. World J Gastroenterol. 2009;15(18):2290-2.

19. Giard JM, Terrault NA. Severe Cholestatic Hepatitis C in Transplant Recipients: No Longer a Threat to Graft Survival. Clin Gastroenterol Hepatol. 2015;13(11):2002-4.

20. Oliver NT, Nieto YL, Blechacz B, et al. Severe hepatitis C reactivation as an early complication of hematopoietic cell transplantation Bone Marrow Transplantation 2016; In press.

21. Torres HA, Chong PP, De Lima M, et al. Hepatitis C Virus Infection among Hematopoietic Cell Transplant Donors and Recipients: American Society for Blood and Marrow Transplantation Task Force Recommendations. Biol Blood Marrow Transplant. 2015;21(11):1870-82.

22. Bosch M, Khan FM, Storek J. Immune reconstitution after hematopoietic cell transplantation. Curr Opin Hematol. 2012; 19(4):324-35.

23. Dick TB, Lindberg LS, Ramirez DD, Charlton MR. A clinician's guide to drug-drug interactions with directacting antiviral agents for the treatment of hepatitis C viral infection. Hepatology. 2016;63(2):634-43.

24. Bodge MN, Culos KA, Haider SN, Thompson MS, Savani BN. Preparative regimen dosing for hematopoietic stem cell transplantation in patients with chronic hepatic impairment: analysis of the literature and recommendations. Biol Blood Marrow Transplant. 2014;20(5):622- 9.

25. Floyd J, Kerr TA. Chemotherapy hepatotoxicity and dose modification in patients with liver disease. In: Drews RE, Lindor KD, eds UpToDate Waltham MA: http://www.uptodate.com. 2016.

26. Rezvani AR, McCune JS, Storer BE, et al. Cyclophosphamide followed by intravenous targeted busulfan for allogeneic hematopoietic cell transplantation: pharmacokinetics and clinical outcomes. Biol Blood Marrow Transplant. 2013;19(7):1033-9.

27. Stine JG, Lewis JH. Hepatotoxicity of antibiotics: a review and update for the clinician. Clin Liver Dis. 2013; 17(4):609-42, ix.

28. Sulkowski MS, Thomas DL, Chaisson RE, Moore RD. Hepatotoxicity associated with antiretroviral therapy in adults infected with human immunodeficiency virus and the role of hepatitis $C$ or B virus infection. JAMA. 2000;283(1):74-80.

29. Viekira pak (ombitasvir p, and ritonavir tablets; dasabuvir tablets). Prescribing Information Revised: 6/2016. AbbVie Inc., North Chicago, IL, USA 